\title{
DESAFIOS E PERSPECTIVAS NA AUDITORIA DE MPCS
}

\author{
V. R. BOTELHO ${ }^{1}$, J. O. TRIERWEILER ${ }^{1}$, M. FARENZENA ${ }^{1}$, G. H. MÜLLER ${ }^{2}$
}

${ }^{1}$ Grupo de Intensificação, Modelagem, Simulação, Controle e Otimização de Processos (GIMSCOP)

Departamento de Engenharia Química, Universidade Federal do Rio Grande do Sul (UFRGS)

${ }^{2}$ TriSolutions Soluções em Engenharia Ltda.

E-mail para contato: \{vivirb, jorge, farenz\}@enq.ufrgs.br

\begin{abstract}
RESUMO - A crescente demanda pela melhoria operacional dos processos aliada ao desenvolvimento da tecnologia da informação tornam a utilização de controladores preditivos baseados em modelos (MPC) uma prática cada vez mais comum na indústria. Contudo, o monitoramento e manutenção destes sistemas ainda é um desafio, principalmente devido às correlações fortes associadas às variáveis que o compõe. Este trabalho visa investigar o potencial de aplicação prática das principais técnicas disponíveis para avaliação de controladores preditivos. Uma revisão teórica foi realizada e algumas técnicas potencialmente aplicáveis industrialmente foram selecionadas e testadas em um sistema de quatro tanques cilíndricos utilizando um controlador preditivo clássico. Constatou-se que as técnicas foram capazes de identificar adequadamente a ocorrência de degradação no desempenho do sistema proposto na maioria dos casos.
\end{abstract}

\section{INTRODUÇÃO}

Controladores preditivos baseados em modelo (MPC) são ferramentas de controle supervisório auxiliares na melhoria operacional dos processos. Estes sistemas usam modelos dinâmicos para predizer o comportamento das variáveis controladas ao longo de um horizonte futuro. Com base no resultado obtido, um algoritmo de otimização calcula as ações de controle que levam o processo as condições operacionais ótimas. A capacidade preditiva destes sistemas é o diferencial que torna o MPC capaz de lidar com dinâmicas complexas, permitindo que os processos permaneçam mais próximos da sua região de máxima produtividade de forma segura e estável. Sua principal vantagem é a possibilidade de lidar com restrições operacionais tanto das variáveis manipuladas quanto controladas, o que o torna a solução mais adequada quando se trabalha com sistemas multivariáveis. 


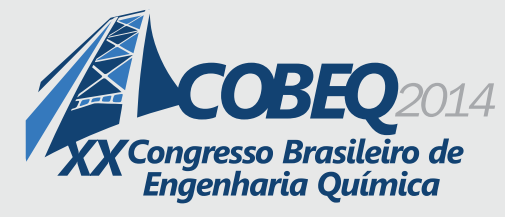

19 a 22 de outubro de 2014

Florianópolis/SC

Embora a avaliação do desempenho de controladores preditivos seja indispensável para a manutenção e estabilidade destes sistemas, este ainda é um tópico sem uma solução completa e totalmente eficiente. A dificuldade associada ao monitoramento e diagnóstico de MPC está vinculada a sua natureza multivariável e suas diferentes formas de implementação. Este trabalho tem o objetivo de avaliar o potencial de aplicação prática das técnicas de auditoria de MPC. Na seção 2, uma revisão teórica das principais técnicas é apresentada. Um estudo de caso referente a um sistema de quatro tanques cilíndricos é apresentada na seção 3 , onde as técnicas de maior viabilidade prática são avaliadas sob diferentes condições de degradação do MPC.

\section{Overview das técnicas de auditoria de controladores preditivos}

Um processo industrial típico contém centenas de malhas de controle as quais interagem entre si. É natural que ao longo do tempo, tais malhas tenham o seu desempenho degradado, uma vez que as mesmas estão sujeitas a fatores externos, como com falhas de sensores, problemas em equipamentos, variabilidade na matéria-prima, mudanças nas características dos produtos, influência sazonal, dentre outros. No caso do controle envolvendo malhas monovariáveis com controladores do tipo PID, diversas técnicas de auditoria são amplamente utilizadas com sucesso no âmbito industrial, a maioria delas baseadas no controlador de variância mínima e nos conceitos apresentados por Harris et al. (1989). No caso dos controladores preditivos, a auditoria torna-se mais complexa dada a natureza multivariável e a capacidade de lidar com restrições das variáveis manipuladas e controladas. A versão mais simples de auditar o desempenho de controladores preditivos é a sua versão quadrada, i.e., quando o número de entradas igual ao número de saídas, sendo que todas as saídas tem um setpoint. Nesta situação o sistema se comporta como sendo um regulatório multivariável e o desempenho de diversos controladores multivariáveis pode ser adotado como referência, sendo os mais usados os baseadas no controlador de variância mínima (MVC) ou variantes como o LQG (Linear Quadratic Gaussian), como os trabalhos de Lee et al. (2008), Harrison et al. (2009) e Zhang et al. (2013). Contudo, muitos autores são desfavoráveis à utilização de LQG/MVC como uma referência para monitoramento de controladores preditivos. Isso porque este controlador pode possuir estrutura e objetivos muito diferentes do MPC industrial, o que o torna uma referência teórica não condizente com os reais objetivos operacionais. (Zhang et al., 2006; Jelali, 2013).

Como alternativa ao MVC/LQG, alguns autores propõe a utilização de referências históricas, as quais consistem em períodos nos quais se sabe de antemão que o controlador está operando da forma desejada. Schafer et al. (2004) propõe uma metodologia a qual visa o monitoramento e avaliação do MPC com base na comparação da função objetivo co MPC com um valor de referência e ainda com o desempenho atingível para o controlador projetado, podendo esta referência ser obtida tanto via LQG quando a partir de uma referência histórica. Qin et al. (2007, 2008-a e 2008-b) propõe uma metodologia com base na investigação da covariância dos períodos de referência e monitorados, visando identificar quais as variáveis controladas que tiveram aumento da variabilidade. Também há uma série de técnicas com enfoque estatístico, a maioria delas fazendo uso de PCA e PLS. É o caso do trabalho de Alghazzawi et al. (2009), cujo método detecta em tempo real, anormalidades na operação do MPC a partir da comparação com um modelo PCA/PLS previamente obtido a partir de um conjunto de dados de referência. O método de Zhang et al. (2006) propõe a criação de uma referência a partir de um modelo PCA de um conjunto de dados históricos com bom desempenho e 


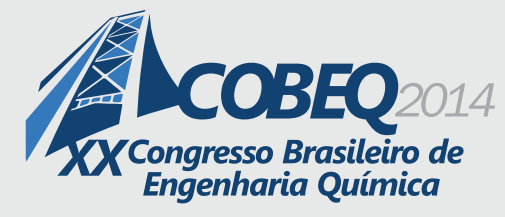

19 a 22 de outubro de 2014

Florianópolis/SC

compara com os dados atuais. O efeito da autocorreção é removido a partir de um filtro ARMA previamente identificado. Alcala et al. (2011) usa PCA e PLS como ferramenta de diagnóstico, sugerindo novos indicadores para monitoramento do desempenho. Existe uma classe de métodos que se baseia na construção de uma rede bayesiana para reconhecer padrões, como o método de Qi et al. (2011). Existem métodos focados em investigar o modelo do processo utilizado pelo MPC, já que este é um dos pontos mais importantes e críticos de um controlador preditivo. Sun et al. (2013) estima que $80 \%$ do tempo gasto no projeto de um MPC seja para obtenção de um modelo, dada a sua importância. É o caso do método de Conner et al. (2005), que usa critério de informação de Akaike para avaliar a necessidade de re-identificação do modelo. Badwe et al. (2009) propõe a avaliação da correlação parcial entre as entradas e resíduos a fim de identificar canais inconsistentes no modelo. Sun et al. (2013) decompõe os resíduos da predição em uma base ortogonal, de modo a identificar quais destes são provenientes de discrepâncias entre modelo e planta. Jiang et al. (2012) apresenta um indicador avaliar a qualidade do modelo, comparando a predição em diferentes níveis. Embora a extensa bibliografia acerca de monitoramento e diagnóstico de controladores preditivos muitas destas técnicas são viáveis apenas na teoria ou em sistemas pouco complexos. Na prática, há alguns critérios desejáveis quando se trata deste tipo de metodologia. Hugo (2002) sugere uma série de critérios desejáveis para um método ideal de auditoria. Baseado nestes critérios, algumas das metodologias disponíveis na literatura foram selecionadas e aplicadas neste trabalho, e serão descritas detalhadamente a seguir.

\subsection{Método de Qin et al. (2007, 2008-a, 2008-b)}

Esta é uma técnica para o monitoramento de controladores multiváriáveis baseada na investigação da variabilidade das variáveis controladas em relação aos seus setpoints. A ideia do método consiste em inicialmente, avaliar a variabilidade global das variáveis controladas, comparando-as com a covariância de um valor de referência histórica. A partir daí investiga-se os efeitos das variáveis sobre esta variabilidade, através da solução do problema de autovalor generalizado apresentado na Equação 1, onde $y_{I}$ e $y_{I I}$ representam a diferença entre a variável controlada e seu setpoint para os dados de referencia e monitorados, respectivamente $\mu$ são os autovalores generalizados e $p$ são os autovetores correspondentes. Autovalores elevados indicam que na direção do autovetor correspondente houve uma degradação no desempenho. Uma inferência estatística é construída para que a análise dos autovalores generalizados seja confiável. Caso o autovalor seja maior/menor que os limites de confiança, identifica-se uma direção de piora/melhora no desempenho. A partir das direções de pior/melhor desempenho subespaços são construídos e a as variáveis controladas associadas a estas direções são identificadas.

$$
\operatorname{cov}\left(y_{I I}\right) p=\mu \operatorname{cov}\left(y_{I}\right) p
$$

\subsection{Método de Badwe et al. (2009)}

Este trabalho propõe uma metodologia para identificar possíveis canais com modelos inconsistentes. Baseia-se na investigação da correlação parcial entre as variáveis manipuladas e os resíduos da predição das variáveis controladas $(\mathrm{CV})$. O autor sugere o uso de correlação parcial como forma de se obter o efeito isolado de cada variável controlada e cada variável manipulada, e obter a 


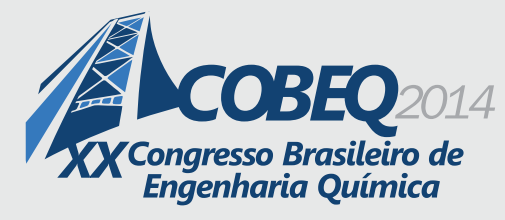

19 a 22 de outubro de 2014

Florianópolis/SC

correlação regular entre estes efeitos, isentos da influência de outras variáveis. A técnica é composta por 3 etapas. Inicialmente deve-se remover o efeito dos distúrbios estocásticos das variáveis manipuladas. Isso é realizado através da identificação de um modelo tipo erro na saída (OE) entre as variáveis manipuladas (MVs) e os setpoints. Em seguida o efeito isolado de cada variável manipulada é obtido, através da identificação de um modelo OE entre cada MV em relação às demais. Por fim, um modelo $\mathrm{OE}$ é identificado entre o resíduo da $\mathrm{CV}$ do canal avaliado em relação as MVs, com exceção daquela que compõe o canal e o efeito do canal é isolado. Dessa forma, a correlação entre as variáveis isoladas pode ser avaliada. A principal adversidade deste método é que, para garantir a confiabilidade nos modelos OE identificados, é necessário que o conjunto de dados avaliado contenha uma quantidade elevada de excitações nos setpoints.

\subsection{Método de Sun et al. (2013)}

Esta é uma técnica baseada no princípio do feedback invariante, no qual as inovações nos distúrbios não são afetadas pelo efeito do feedback do controlador, permitindo que os distúrbios estocásticos sejam estimados a partir de dados em malha fechada. $\mathrm{O}$ autor sugere que esta estimação seja realizada através da identificação de um modelo ARX de elevada ordem entre as variáveis controladas e os setpoints. A qualidade preditiva dos modelos é avaliada através da comparação dos resíduos de predição one-step-ahead das variáveis controladas. A partir da relação entre o ruído estimado pelo modelo $\operatorname{ARX}(e(k))$ e os resíduos de predição do modelo $\left(e^{0}(k)\right)$ é possível avaliar quantitativamente qual parcela do erro de predição é efetivamente decorrente de problemas no modelo ou causada por distúrbios determinísticos. Sun et al. (2013) sugere um indicador que leva em conta a importância das variáveis, denominado índice de qualidade do modelo (MQI), conforme a Equação 2, onde $Q$ é a matriz de pesos das variáveis controladas. Este indicador deve assumir valores entre 0 e 1 de modo que, $\mathrm{MQI}=1$ representa um modelo perfeito.

$$
M Q I=\frac{\sum_{k=1}^{N} e^{0}(k)^{T} Q e^{0}(k)}{\sum_{k=1}^{N} e(k)^{T} Q e(k)}
$$

\section{Estudo de caso - Planta de Quatro Tanques Cilíndricos}

\subsection{Descrição do sistema}

A planta de quatro tanques cilíndricos é um exemplo capaz de ilustrar diversos conceitos de controle multivariável, dada sua capacidade de mudança na posição do zero de transmissão. O processo é composto por quatro tanques cilíndricos conectados de acordo com a Figura 1. A vazão de fluído é dada pelas voltagens das bombas $v 1$ e $v 2$ e a razão que migra para cada tanque pela abertura das válvulas $x 1$ e $x 2$. Dependendo da posição das válvulas o processo pode ser caracterizado por um sistema de fase mínima (zero de transmissão localizado no semi-plano esquerdo) ou fase não-mínima (zero de transmissão localizado no semi-plano direito). Maiores detalhes sobre o modelo do processo podem ser encontrados em Johansson (2000). 


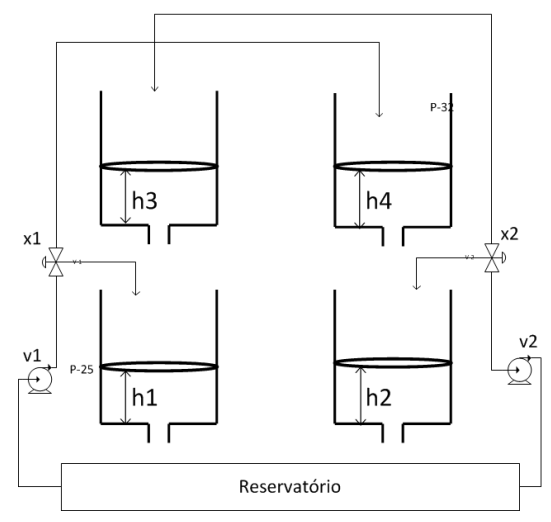

Figura 1 - Planta de quatro tanques cilíndricos.

Um controlador preditivo foi projetado para este sistema no Matlab. O objetivo do sistema é controlar os níveis dos tanques inferiores através da manipulação das voltagens das bombas. Os parâmetros de sintonia foram configurados de acordo com a metodologia RPN desenvolvida por Trierweiler et al. (2001). Dados de processo foram artificialmente gerados a partir da simulação do sistema considerando variações nos setpoints das variáveis manipuladas e com adição de ruído branco de magnitude $1 \%$ nas variáveis controladas. Diferentes cenários foram gerados, os quais simulam diferentes fontes de degradação no MPC, podendo ser estas no modelo e na sintonia, conforme ilustra a Tabela 1 .

Tabela 1- Descrição dos Cenários avaliados

\begin{tabular}{|c|c|c|c|c|c|}
\hline Cen. & Descrição & Cen. & Descrição & Cen. & Descrição \\
\hline 0 & -- & 6 & $\begin{array}{c}\text { Resposta mais lenta no } \\
\text { canal } v_{1} X h_{1}=4 X\end{array}$ & 12 & Cenário 4 e Cenário 5 \\
\hline 1 & $\begin{array}{c}\text { Ganho do canal } \\
v_{1} X h_{1}=-1 X\end{array}$ & 7 & $\begin{array}{c}\text { Resposta mais rápida } \\
\text { no canal } v_{1} X h_{1} \\
=0.5 X\end{array}$ & 13 & Cenário 8 Cenário 10 \\
\hline 2 & $\begin{array}{c}\text { Ganho do canal } \\
v_{1} X h_{2}=0.5 X\end{array}$ & 8 & $\begin{array}{c}\text { Tempo morto no canal } \\
v_{2} X h_{2}=100 s\end{array}$ & 14 & $\begin{array}{c}\text { Ruído branco mais } \\
\text { intenso }=10 X\end{array}$ \\
\hline 3 & $\begin{array}{c}\text { Ganho do canal } \\
v_{1} X h_{2}=1.2 X\end{array}$ & 9 & $\begin{array}{c}\text { Tempo morto no canal } \\
v_{2} X h_{1}=40 s\end{array}$ & 15 & $\begin{array}{c}\text { Removido parâmetro } \\
\text { de sup. de movimento }\end{array}$ \\
\hline 4 & $\begin{array}{c}\text { Ganho do canal } \\
v_{1} X h_{2}=2 X\end{array}$ & 10 & $\begin{array}{c}\text { Oscilação em } \\
h_{1}=h_{1}+\sin (0.005 t)\end{array}$ & 16 & $\begin{array}{c}\text { Aumento no parâmetro } \\
\text { de sup. de movimento }\end{array}$ \\
\hline 5 & $\begin{array}{c}\text { Resposta mais lenta no } \\
\text { canal } v_{1} X h_{1}=2 X\end{array}$ & 11 & $\begin{array}{c}\text { Decréscimo em } \\
h_{2}=h_{2}-t / 10000\end{array}$ & 17 & Alteração no peso $h_{2}$ \\
\hline
\end{tabular}

\subsection{Resultados e discussões}

As metodologias descritas na seção 2 foram aplicadas no estudo de caso proposto. Inicialmente, avaliou-se o método de Sun et al. (2013). Pode-se constar a partir da Figura 2 que, na maioria dos 


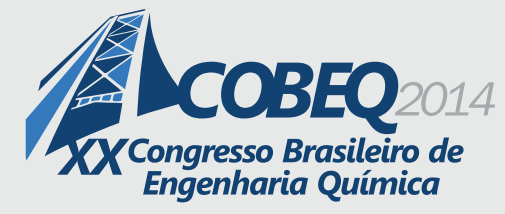

casos ele é capaz de capturar os problemas de modelagem nas variáveis controladas, indicando decréscimo no indicador na presença de incompatibilidades no modelo ou distúrbios determinísticos (cenários de 1 à 13). Já na presença de distúrbios estocásticos (cenário 14) e degradação da sintonia (cenários de 15 a 17) o método corretamente não atribui a piora no desempenho a problemas de modelagem. Observa-se ainda que, sob condições mais brandas de degradação (cenários 3, 5 e 11), a sensibilidade do método é baixa. Contudo, constatou-se que, de fato, tais cenários não promoveram modificações significativas no desempenho do sistema.

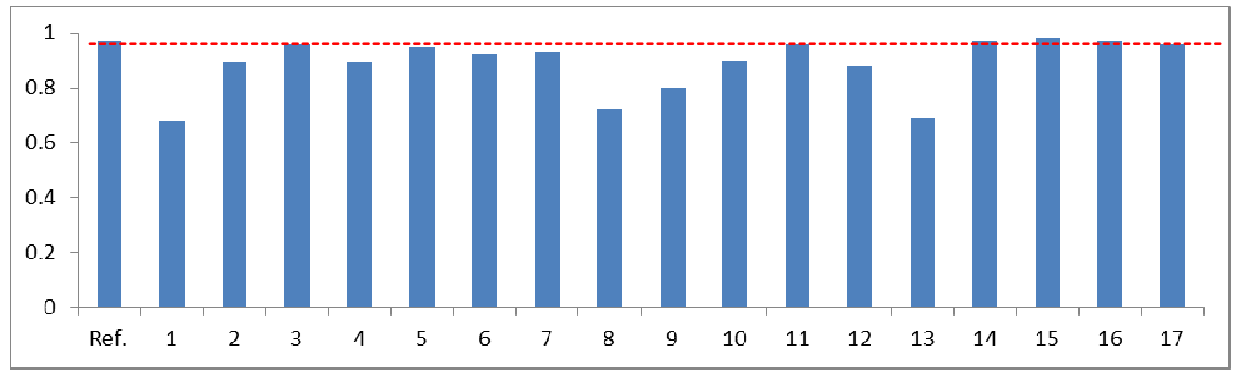

Figura 2 - Resultados do método de Sun et al. (2013): MQI para cada cenário.

O método de Qin et al. (2007, 2008-a e 2008-b) indica de forma adequada a variável controlada teve seu desempenho degradado, independentemente se esta é causada por problemas na modelagem ou na sintonia do controlador. Contudo, o método é pouco sensível à maioria dos problemas, indicando piora no desempenho apenas nos cenários 2,9,14, 16 e 17. Na prática, isso significa que, ainda que o controlador avançado trabalhe com inconsistências nos modelos, o impacto desta variável no seu desempenho, em termos de variabilidade, nem sempre é significativo, não justificando uma nova identificação ou revisão da sintonia.

O método de Badwe et al. (2009) tem como principal objetivo apontar a existência de problemas em canais específicos do modelo do processo com na análise da correlação parcial. Constata-se que o método é capaz de apontar canais corrompidos no modelo do processo, caso estes sejam significativos. Mesmo quando há mais de uma causa prejudicando o desempenho, o método foi capaz de detectar adequadamente, como nos casos dos cenários 12 e 13. Ele é insensível a discrepâncias pequenas no modelo do processo (cenário 3). Ele também se mostrou adequado quando há excesso de ruído, distúrbios não medidos ou problemas de sintonia (cenários 10, 11 e de 14 a 17), não apontando nenhum canal do modelo como responsável pela degradação. Dessa forma, pode-se concluir que o método se mostrou adequado para avaliação de controladores preditivos. A Figura 3 exemplifica os resultados obtidos, ilustrando correlação parcial elevada para os canais com modelo degradado (Figura 3-a e 3-b) e correlação inexistente quando o problema no desempenho não é causado pelo modelo (Figura 3-c). É importante ressaltar a necessidade da existência de perturbações suficientes nas variáveis controladas e manipuladas para o sucesso da metodologia, caso contrário, ela leva a interpretações equivocadas. 

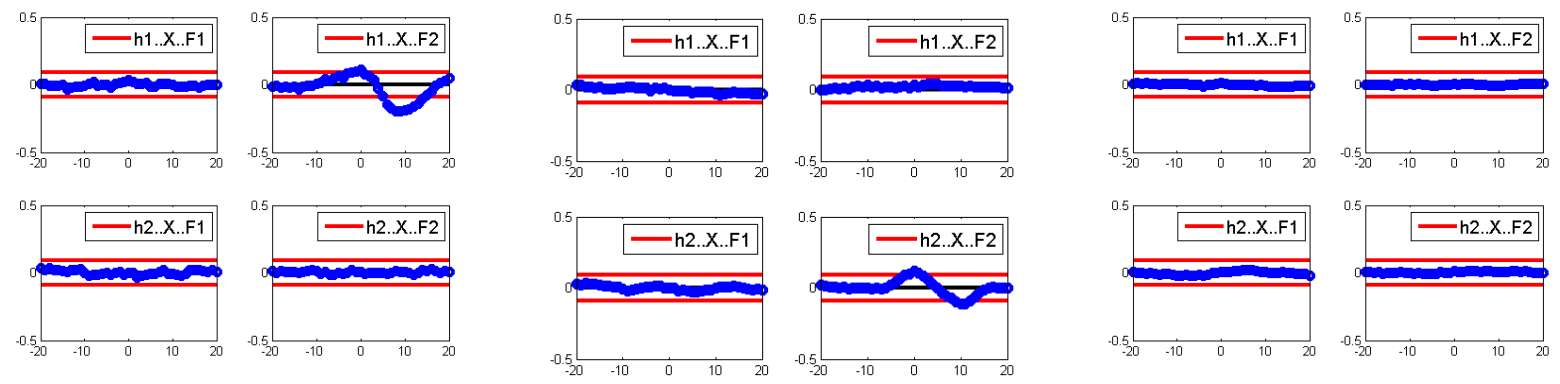

(a)

(b)

(c)

Figura 3 - Resultados do método de Badwe et al. (2009) para os cenários 9 (a), 13(b) e 16(c):

Gráficos de correlação parcial cruzada

\section{Conclusões}

Neste trabalho um estudo teórico das técnicas de auditoria de MPC foi realizado e foram selecionadas técnicas potencialmente aplicáveis em sistemas industriais. Todas elas indicaram bons resultados para o estudo de caso proposto. O método de Sun et al. (2013) indicou adequadamente as variáveis controladas com problemas de modelagem ou sendo deteriorada pela presença de distúrbios determinísticos. O método de Badwe et al. (2009) foi capaz de apontar adequadamente quais os canais do modelo estavam com modelos inconsistente, indicando correlação parcial elevada nestes casos. O método de Qin et al. (2007, 2008-a e 2008-b) indicou corretamente as variáveis responsáveis pela degradação do desempenho no controlador, assim como se mostrou um indicador útil na quantificação do impacto dos problemas de modelagem e sintonia, já que foi insensível a estes quando eles não pioravam o sistema de maneira significativa.

Embora os controladores preditivos possuam a mesma fundamentação teórica, a maioria das aplicações industriais leva em conta uma série de considerações práticas, como por exemplo, existências de camada de otimização dinâmica simplificada, sistemas com graus de liberdade insuficientes, variáveis operando por faixas, etc. Muitas destas implementações não podem ser facilmente formuladas sob uma estrutura matemática unificada, dificultando a aplicação das técnicas de monitoramento e diagnóstico, já que estas, em sua maioria, são desenvolvidas embasadas nos conceitos tradicionais de controladores preditivos. Sendo assim, a próxima etapa deste trabalho consiste na incorporação de alguns destes aspectos práticos e utilização de um controlador comercial para avaliar as técnicas e identificar formas de flexibilizá-las a fim de permitir a incorporação de tais aspectos no processo de avaliação.

\section{Referências}

ALCALA, C.; QIN, J. Analysis and generalization of fault diagnosis methods for process monitoring. Journal of Process Control v. 21, p. 322-330, 2011.

ALGHAZZAWI, A.; LENNOX, B. Model predictive control monitoring using multivariate statistics. Journal of Process Control, v. 19, p. 314-327, 2009. 
BADWE, A.; GUDI, R.; PATWARDHAN, R.; SHAH, S.; PATWARDHAN, S. Detection of model-plant mismatch in MPC applications. Journal of Process Control, v. 19, p. 1305-1313, 2009.

CONNER, J.; SEBORG, D. Assessing the Need for Process Re-identification. Ind. Eng. Chem. Res., v. 44, p. 2767-2775, 2005.

HARRIS, T. J.; Assessment of Control Loop Performance. The Canadian Journal of Chemical Engineering, v. 67, p. 856-861, 1989.

HARRISON, C.; QIN, S. Minimum variance performance map for constrained model predictive control. Journal of Process Control, v.19, p.1199-1204, 2009.

HUGO A.; Process Controller Performance Monitoring and Assessment. Control Arts Inc, 2002.

JELALI, M. Control Performance Management in Industrial Automation: Assessment, Diagnosis and Improvement of Control Loop Performance. Londres: Editora Springer, 2013.

JIANG, H.; SHAH, S.; HUANG, B. WILSON,B., PATWARDHAN, R.; SZETO, F. Model analysis and performance analysis of two industrial MPCs. Control Engineering Practice. v. 20, p. 219-235, 2012.

JOHANSSON, K. The Quadruple-Tank Process: A Multivariable Laboratory Process with an Adjustable Zero. EEE Transactions on Control Systems Technology, v. 8, 2000.

LEE, K. H.; HUANG, B.; TAMAYO, E. Sensitivity analysis for selective constraint and variability performance assessment of industrial MPC. Control Engineering Practice, v. 16, p. 1195-1215, 2008.

QI, F.; HUANG, B. Bayesian methods for control loop diagnosis in the presence of temporal dependent evidences. Automatica, v. 47, p. $1949-1356,2011$.

QIN, J.; YU, J. Recent developments in multivariable controller performance monitoring. Journal of Process Control, v. 17, p. 221-227, 2007.

QIN, J.; YU, J. Statistical MIMO controller performance monitoring. Part I: Data-driven covariance benchmark, v. 18, p. 277-296, 2008.

QIN, J.; YU, J. Statistical MIMO controller performance monitoring. Part II: Performance diagnosis, v. 18, p. 297-319, 2008.

SCHAFER, J.; CINAR, A. Multivariable MPC system performance assessment, monitoring, and diagnosis. Journal of Process Control, v. 14, p. 113-129, 2004.

SUN, Z.; QIN, J.; SINGHAL, A. MEGAN, L. Performance monitoring of model-predictive controllers via model residual assessment. Journal of Process Control, v. 23, p. 473-482, 2013.

TRIERWEILER, J. ;FARINA, L; DURAISK, R. RPN Tuning Strategy for Model Predictive Control. Preprints of DYCOP , p. 283-288, 2001.

ZHANG, Q.; SHAYUAN, L. Performance Monitoring and Diagnosis of Multivariable Model Predictive Control Using Statistical Analysis. . Chinese Journal of Chemical Engineering, v. 14, p. 207-215, 2006. 